\title{
Las TIC en la educación infantil: una revisión sistemática de las políticas públicas de México y Costa Rica
}

\author{
ICT in early childhood education: a systematic review \\ of public policies in Mexico and Costa Rica
}

RECIBIDO 9/4/2021 ACEPTADO 25/5/2021 PUBLICADO 1/12/2021

\author{
(iD) Martha Patricia Astudillo Torres \\ Departamento de Desarrollo Curricular, Universidad Autónoma de Chiapas, Méxicoa \\ patricia.astudillo@unach.mx \\ iD Florlenis Chévez Ponce \\ Ministerio de Educación Pública, Dirección de Gestión y Evaluación de la Calidad, México \\ florlenis.chevez.ponce@mep.go.cr \\ Yesenia Milagros Oviedo Vargas \\ Ministerio de Educación Pública, Dirección de Gestión y Evaluación de la Calidad: Departamento de Evaluación Académica y Certificación, México \\ yessenia.oviedo.vargas@mep.go.cr
}

\section{RESUMEN}

La investigación tiene como objetivo analizar las políticas públicas acerca de la integración de las TIC en el proceso de enseñanza y aprendizaje de la población infantil, que cursa en preescolar y educación primaria, en México y Costa Rica. La metodología que se utiliza es el análisis de contenido, la cual se enfoca en diferentes fuentes institucionales referidas a la población determinada en los países estudiados. Por tanto, se considera información sistemática, objetiva, replicable y válida sobre el uso de las TIC en la infancia que han sido categorizadas mediante las unidades de análisis (segmentos expuestos en los mensajes escritos y visuales establecidos en clasificaciones y subclasificaciones de los cuales se derivan los hallazgos de la investigación), que permiten evidenciar la importancia de las TIC en la población infante, subrayando que la niñez debe considerarse como personas que pueden participar activamente en su proceso de aprendizaje. Otro de los elementos a destacar es que los Estados deben procurar el establecimiento de políticas públicas para sentar las bases del avance tecnológico en las poblaciones infantiles. Asimismo, se enfatiza que las políticas públicas deben tener un seguimiento por parte de los gobiernos, procurando instituir un proceso de continuidad para obtener la seguridad de que estas se cumplan en todas sus dimensiones.

PALABRAS CLAVE infancia, tecnología de la información, educación, análisis de contenido.

\section{ABSTRACT}

The presente research aims to analyze public policies about the integration of ICT in the teaching and learning process of the child population, who attend preschool and primary education, in Mexico and Costa Rica. The methodology used is content analysis, which focuses on different institutional sources referring to the specific population in the countries studied; Therefore, it is considered systematic, objective, replicable and valid information on the use of ICT in childhood that has been 
categorized through the units of analysis - segments exposed in the written and visual messages established in classifications and sub-classifications, of which derive the research findings - which allows to demonstrate the importance of ICT in the infant population, stressing that children should be seen as people who can actively participate in their learning process; Another element to be highlighted is that the States must endeavor to establish public policies to lay the foundations for technological advance in child populations. Likewise, it is emphasized that public policies must be followed up by governments, seeking to institute a continuity process to obtain assurance that these are met in all their dimensions.

KEYWORDS childhood, information technology, education, content analysis.

\section{INTRODUCCIÓN}

La enseñanza y el aprendizaje que se lleva a cabo en los diferentes niveles implican el establecimiento de políticas educativas tendientes a guiar el avance de la población estudiantil según la escolaridad que curse.

Uno de los procesos que cobra relevancia, en los actuales lineamientos, está referido a la infancia. Por consiguiente, en esta investigación se toman como pilares fundamentales los diferentes documentos que apuntan hacia el desarrollo de la educación de la niñez, en procura de que esta considere los elementos que aportan las Tecnologías de la Información y la Comunicación (TIC) en el proceso educativo. Por ende, el planteamiento que se genera en este trabajo es considerar a la niñez "como una categoría, social e históricamente construida, utilizada para designar al grupo social que conforman las/los infantes" (Duek, \& Benitez, 2018, p. 124). Es decir, bajo esta óptica, la niñez se considera como sujeto de derechos, que tiene su propio desarrollo y, por lo cual, no puede entenderse como objeto pasivo.

En Costa Rica, desde uno de los planteamientos de la Política Educativa, se señala que "los procesos educativos propiciarán ambientes de aprendizaje novedosos, en los cuales la tecnología potencie la creatividad y el conocimiento e incorpore, desde la primera infancia, formas de aprendizaje activas y participativas" (Ministerio de Educación Pública, 2017, p. 14). Por lo tanto, el sistema educativo tiene como eje fundamental la continuidad de conectividad y el uso de las TIC, cuyo propósito es cerrar la brecha digital.

En el caso de México, la política educativa se genera como un flujo articulado, regulado y direccionado de bienes, servicios y transferencias que se moviliza desde el Estado para garantizar el derecho a la educación de la niñez y adolescentes, desde el nivel de preescolar hasta su graduación de la educación media superior (Instituto Nacional para la Evaluación de la Educación, 2018).

Estas premisas, en los países en los que se centra la investigación, permiten que la tecnología sea un elemento fundamental en el desarrollo educativo de la niñez. No obstante, se identifica un problema fundamental: el acceso digital se muestra como una línea divisoria, puesto que, millones de niños y las niñas que podrían entrar con mayores ventajas al tener acceso a las tecnologías, no están en contacto con ella.

\subsection{Antecedentes: las TIC en la educación infantil}

Las TIC como medio para llevar a cabo el proceso educativo es un tema que ha sido estudiado desde diversos contextos académicos. Sin embargo, resulta indispensable analizar la integración de estas herramientas tecnológicas a la educación, ahora que el proceso de educación formal en todos los niveles se realiza desde 
la virtualidad total debido a la crisis sanitaria originada por la enfermedad COVID-19. De esta manera, las TIC han constituido la plataforma tecnológica que ha permitido la continuación de la enseñanza y el aprendizaje desde casa. Por ello, es relevante tomar en cuenta las voces de diversos autores que han estudiado la temática, centrada en la educación infantil, el cual se constituye en el tema de este trabajo.

En este sentido, Ruiz, \& Hernández (2018) señalan que el profesorado considera las TIC como potenciadoras del aprendizaje en la población infantil. No obstante, son pocos los docentes que usan las TIC de manera cotidiana en su quehacer con infantes. La mayor parte de los profesores demandan más cualificación para el uso de herramientas tecnológicas en clases y señalan que la dotación tecnológica de los centros educativos es insuficiente. Asimismo, Briceño et al. (2018) evidencian que, pese a los cambios y la convicción sobre el uso de las TIC de profesoras de preescolar, algunas de ellas hacen un uso restringido de las mismas en la planeación y elaboración de material didáctico (Cabero, 2007; Coll, 2009), especialmente por falta de conocimiento en el manejo de programas que puedan serles útiles para este fin y la falta de apropiación de dispositivos tecnológicos que puedan contribuir en el proceso de enseñanza y aprendizaje. Asimismo, debido a la incorporación de las TIC en la niñez, se plantea una perspectiva de estudio que parte de las teorías de Piaget y Vygotsky en las que se aprovecha el juego digital como estrategia de aprendizaje para las infancias, así el adulto es el "andamiaje" fundamental. Igualmente, se manifiesta la importancia del ambiente positivo familiar y escolar en el uso de las tecnologías para que la zona de desarrollo próximo promueva la motivación en el niño y maximice su experiencia (Siraj, \& Romero, 2017).

En cuanto a la población de infantes vulnerables, Atar (2020) indica que el uso de la tecnología en los entornos de atención y educación de la primera infancia contribuye positivamente al desarrollo de los niños y las niñas. Sin embargo, esta posición genera puntos de vista contradictorios, ya que las circunstancias individuales dependen en gran medida de dónde nace y se cría un niño. Específicamente, en niños y niñas de Nigeria, el uso de las TIC en la educación ha traído como consecuencia tensiones entre las innovaciones sociales y las implicaciones socioculturales, observándose que el empleo de las TIC en la educación infantil de este país se sitúa dentro de una clasificación tripartita; 1) como una verdadera herramienta para lograr el pensamiento innovador y creativo en la niñez; 2) como un instrumento eficaz para difundir ideas globalizadas, y 3) como distorsiones de los valores socioculturales que están arraigados en el aprendizaje y el desarrollo de la infancia culturalmente sensible.

Así también, debido al incremento y llegada a las aulas escolares de alumnado procedente de países distintos, aunado al aumento de una sociedad ampliamente multicultural, las necesidades que esta situación demanda han de trabajarse desde edades tempranas en el sistema educativo, para acercar a los alumnos al entendimiento mutuo de la sociedad multicultural. En ella, el punto de referencia, ineludiblemente, son las TIC, que han posibilitado un trabajo innovador de los contenidos didácticos, resaltando una mejora de la motivación, interés e implicación de los alumnos infantes (Rodríguez et al. 2019).

Como se ha expuesto, son dos vertientes las que giran en torno a la integración de las TIC en la educación infantil, la primera, relacionada con la insuficiente infraestructura tecnológica en los centros educativos y la escasez de conocimientos tecnológicos y pedagógicos de los docentes para la inclusión de las TIC en las clases con los infantes. La segunda perspectiva, más favorable, alude a la facilitación del proceso educativo cuando se emplean adecuadamente recursos tecnológicos, con orientación didáctica, para desarrollar los contenidos curriculares con población infantil, incluyendo población multicultural y vulnerable. 


\subsection{Las políticas educativas sobre la inclusión de las TIC en la educación de las infancias}

El uso de las tecnologías en el mundo tiene una afectación sobre las actividades diarias de los seres humanos en las diferentes etapas de su vida. Los individuos ejercen una ciudadanía planetaria que les permite estar en contacto con gran cantidad de personas y elementos físicos y digitales, en relación con las tecnologías de la información y de la comunicación debe y puede tener su inicio en la infancia, "ya que el conocimiento de otras culturas a través de las TIC supone la adquisición de hábitos conductuales que tienen que ver con la tolerancia y el respeto a los demás" (Soto, 2007, p.8).

Por otra parte, como expone el Fondo de Naciones Unidas para la Infancia (UNICEF), la niñez vulnerable puede correr un mayor peligro de sufrir algún tipo de daño, incluida la pérdida de privacidad (UNICEF, 2017). Es, por tanto, una necesidad conocer acerca de esfuerzos en materia política que permita legislar a favor de la niñez del mundo.

\subsection{Políticas internacionales sobre tecnología en el proceso educativo en las infancias}

El considerar las políticas internacionales en lo que respecta a las TIC requiere mirar los diferentes elementos interventores, de manera que, como afirma el Ministerio de Educación Pública (MEP), el uso de las TIC logre transformar los "factores como las políticas locales, los intereses económicos, el índice de desarrollo humano, los programas educativos, el acceso a energía e internet, la organización local y los movimientos sociales, entre otros" (MEP, 2017, p. 10).

Al respecto, UNICEF (2017) indica que existe la necesidad de establecer programas en el desarrollo de estrategias para evidenciar "las capacidades necesarias para mediar de manera positiva en el uso de las TIC por parte de la niñez, en lugar de simplemente restringirlo” (p.31) Así también, existen esfuerzos que las organizaciones realizan para brindar lineamientos en el abordaje de las tecnologías en el mundo, se tiene que en el año 2006 el Fórum Mundial de la Sociedad de la Información se convirtió en el mecanismo global para la implementación de los acuerdos de Ginebra y Túnez "y en una plataforma para la participación e identificación de tendencias en el área de la sociedad de la información” (Rueda, 2018, p.11).

Las políticas mundiales están más enfocadas en la accesibilidad de las tecnologías y regulación de su uso por parte de la niñez, que en las potencialidades que se pueden impulsar a partir del contacto de la infancia con la era digital. La UNICEF (2017) en este aspecto apunta a que la tecnología es un elemento que permite el cambio de diversas las situaciones de la niñez que ha quedado retrasada por motivos de "la pobreza, la raza, el origen étnico, el género, la discapacidad, el desplazamiento o el aislamiento geográfico al conectarlos a numerosas oportunidades y dotarles de las aptitudes que necesitan para tener éxito en un mundo digital" (p.1).

La niñez alrededor del mundo se conecta a internet para realizar diversas acciones, como por ejemplo estudiar, tener entretenimiento o para comunicarse con otras personas, pero no en igualdad de condiciones; esa conectividad va a depender de las políticas de cada nación, es así, como se evidencian modificaciones "en las relaciones entre pares al traspasar los muros de la escuela, lo que puede resultar positivo o dañino para los miembros de la comunidad escolar. Es necesario reflexionar acerca del papel de la escuela" (UNICEF, 2014, p. 9) y, además, revisar en materia de política educativa qué se está realizando, pues afecta directamente a cada individuo a lo largo de su vida personal y profesional. Este ineludible enlace entre lo 
tecnológico y educativo supone un desafío mayor y es la "dificultad de implantar" a la educación elementos que le son extraños, que no surgen ni se desarrollan dentro de los sistemas educativos y que, por tanto, no se instalan en ellos de manera" (Carneiro et al. 2018, p.30).

\subsection{Políticas regionales sobre las tecnologías en el proceso educativo en las infancias en América Latina}

En Latinoamérica, los esfuerzos para un establecimiento de políticas regionales relacionadas con el uso de las tecnologías en los procesos educativos de las infancias aún requieren mayor dinamismo, pues en la agenda 2030 las TIC y la infancia se visualizan desde la escuela. Siguiendo al Sistema de Información de Tendencias Educativas en América Latina (SITEAL), en casos como "Brasil, Argentina, Honduras, El Salvador, Colombia y Guatemala ya se tienen normas para el eje de la Educación y las TIC, pero hace más referencia a elementos generales que aspectos medulares de la infancia" (SITEAL, 2019, p.3). Se tiene que en países como Brasil, Argentina y Uruguay "el Estado ha desempeñado un papel central como protagonista e inversor en la infraestructura tecnológica” (Rueda, \& Franco, 2018, p. 11) con fines de cobertura de la población escolar.

Asimismo, se relaciona el uso de tecnologías con los aprendizajes escolares y no se considera el disfrute de la niñez, el esparcimiento y su derecho al libre acceso, por lo que las políticas están más afines con el desarrollo de habilidades digitales, sin embargo; se plasma que el acceso que se da del uso de las TIC, no llena las expectativas, por lo que se requiere realizar esfuerzos adicionales para mejorar dicha experiencia y entregar más herramientas, las que debieran estar enfocadas no solo a los fines educativos sino también a preparar a los estudiantes para las realidades cotidianas que van más allá de la escuela (Pavez, 2014).

\subsection{Políticas educativas en la educación de las infancias en México y Costa Rica}

En lo que compete a estos dos países, el uso de las tecnologías por parte de la niñez, no se escapa a la realidad que presenta el resto de América Latina, un enfoque político centrado en la cobertura, un Estado regulador del acceso al área digital y un gran esfuerzo por la creación de infraestructura tecnológica en el país.

En el caso de México, el uso de las TIC en la infancia conlleva el doble reto: construir una política pública para la primera infancia y proyectar los pasos a realizar más allá de los infantes, como lo es la etapa de la niñez de los 6 años a los 12 en materia tecnológica, que básicamente son los años escolares. En un país donde "más de un tercio de los usuarios de medios digitales son niños y jóvenes" (Espinoza, \& Rodríguez, 2017, p.17), no existe una política clara acerca del uso de las TIC y la población escolar, aunque se realizan esfuerzos y propuestas aisladas, se apunta hacia la cobertura.

Al respecto se tiene que "el gasto público destinado a primera infancia es insuficiente; tampoco se sabe cuánto se gasta ni en qué de manera específica para este grupo etario" (El diálogo, 2019, p.4) por lo que el tema de las tecnologías de información aún no se tiene claro. Los datos de pobreza o problemáticas sociales y económicas sí tienen registro, pero existe ausencia de legislación y política curricular en lo que respecta al uso de las TIC y la inclusión de la niñez. En este país, es la empresa privada quien ha tomado un rol protagónico en la expansión de infraestructura tecnológica.

El gobierno mexicano, por su parte, señala que los recursos educativos como libros, cuadernos, pizarrones, computadoras, proyectores, pantallas, entre otros, tienen repercusión positiva cuando cuentan con un 
acompañamiento pedagógico adecuado a las necesidades e intereses de la población estudiantil en el logro de mejores aprendizajes. Sin embargo, no siempre se tiene información confiable sobre el uso de los recursos educativos por parte de ciertos grupos de la población, generalmente aquellos que presentan un mayor rezago. En consecuencia, se requiere desarrollar estrategias que aseguren la distribución de estos recursos y su uso entre los grupos más vulnerables de la población, ya que eliminar brechas no es solo una cuestión de acceso sino de aprovechamiento. Es decir, disminuir la brecha digital no implica solamente dar acceso a las tecnologías a aquellos menos favorecidos, sino prepararlos para contar con los conocimientos y las habilidades necesarias para adaptarse al cambio tecnológico y utilizarlas para el fortalecimiento de sus aprendizajes (Diario Oficial de la Federación -DOF-, 2020).

Igualmente, es de suma importancia para el gobierno asegurar la relevancia y la pertinencia de la educación en todos los tipos, niveles y modalidades, buscando la mejora continua, por lo que se requiere no solo de grandes esfuerzos de los principales actores del Sistema Educativo Nacional, sino también de un cambio de paradigma que repercuta de manera sustantiva en la forma en que las niñas, niños, adolescentes y jóvenes perciben el mundo y aprenden de él. Por ello, los planes y programas de estudio serán revisados y adecuados a las necesidades y desafíos actuales para lograr una educación integral y de calidad desde la primera infancia hasta la educación superior, que comprenda, entre otros campos, la salud, el deporte, la literatura, el arte, la música, el inglés, el desarrollo socioemocional, así como la promoción de estilos de vida saludables, de la educación sexual y reproductiva, del cuidado al medio ambiente y del uso de las tecnologías de la Información, Comunicación, Conocimiento y Aprendizaje Digital (TICCAD).

Con base en este objetivo, se establece una estrategia prioritaria para instrumentar métodos pedagógicos innovadores, inclusivos y pertinentes, que fortalezcan los procesos de enseñanza y aprendizaje orientados a mejorar la calidad de la educación que recibe la niñez, adolescentes y jóvenes. Se propone, para ello, el apoyo para el acceso y utilización de las TICCAD en los procesos de la vida cotidiana con una perspectiva crítica de los contenidos y materiales disponibles en medios electrónicos, plataformas virtuales y redes sociales (DOF, 2020).

En el caso de Costa Rica, "a través del Ministerio de Educación Pública (MEP) se ha realizado importantes esfuerzos para incorporar la informática a la Enseñanza General Básica a través del Programa Nacional de Informativa Educativa (PRONIE), con la colaboración de la Fundación Omar Dengo (FOD)” (Calderón et al., 2013, p.5).

Es preciso destacar que estos programas, sus alcances y resultados no han sido evaluados oportunamente y con la regularidad requerida, ya que "igual que muchos países en el mundo, Costa Rica carece de sistemas de monitoreo y evaluación de los procesos de incorporación de las TIC en la educación que permita conocer el resultado de los esfuerzos nacionales en ese sentido" (Cuevas, \& Núñez, 2016, p.399).

Este país sí posee una política educativa en lo que respecta a la primera infancia, pero aún está por concretar lo referente al uso de las tecnologías de información, y por otra parte resulta una tarea pendiente la política curricular en materia digital y su afectación en los diferentes niveles de organización del MEP.

\subsection{El paradigma sociocultural como fundamento en la inclusión de las TIC en la educación de infantes}

El análisis de contenido referido a las políticas que convergen en el uso de las TIC en los procesos de enseñanza y aprendizaje desde la infancia, requieren de un abordaje teórico que sustente las relaciones que se evidencian en el desarrollo de los contenidos curriculares y habilidades que logra la niñez en el contexto educativo. 
Es así como la fundamentación teórica se basa en los postulados de la Teoría Sociocultural de Vygotsky, la cual está referida a la participación que manifiesta la niñez de acuerdo con el ambiente que la rodea. En este trabajo investigativo se acentúa la relación de los elementos inmersos en el ámbito de la educación con el uso de las TIC.

El principal componente considerado se refiere a la zona de desarrollo próximo (ZDP) en el binomio desarrollo y aprendizaje, y es a partir de aquí, que se visualiza que existen dos niveles de desarrollo: en primer lugar, lo que alcanza el niño en forma individual y, en segundo lugar, lo que el niño logra alcanzar desde el desarrollo potencial basado en la ayuda de un adulto (Vygotsky, 1978). Es decir, estos dos niveles se dirigen a establecer un posicionamiento en la mediación pedagógica en procura de alcanzar una transmisión e interiorización de los elementos culturales en un ambiente y un momento determinado. La conceptualización de los papeles relacionados con los procesos individuales y culturales y la orientación de cómo pensar acerca de los conocimientos culturales en el desarrollo humano, que son vistos desde una perspectiva sociocultural. En este sentido, “ni el bagaje cultural, ni el genético, se pueden dar por aparte, ambos forman una combinación indivisible, que limita en gran parte la clara definición de cuál es la mayor influencia en el desarrollo de la personalidad" (Smith, 1991, p.5).

Aunado a lo anterior, el autor, indica que esto le permite tener un panorama más amplio de todos los elementos que confluyen en la naturaleza del desarrollo cultural. Desde la posición de Rogoff (1993), es fundamental hablar de desarrollo natural de la cultura humana, en el sentido de que el desarrollo humano es un proceso cultural, donde el ser humano se define de acuerdo con su participación en la construcción de la cultura, de manera que se visualice como un todo, desde lo biológico y lo cultural. Al respecto, lo cultural "no es sencillamente colecciones de gente que comparte un lenguaje y tradición histórica común, sino, que se compone de instituciones que especifican, de forma más concreta, qué funciones tiene la gente y qué estatus se les otorga" (Bruner, 1997, p. 48).

Por tanto, es preciso hablar del desarrollo natural de la cultura humana, tal y como lo indica Rogoff (2003), al estudiar varios aspectos del desarrollo como la crianza de los hijos, las diferencias que se establecen en la sociedad en cuanto al género, la disciplina de los padres, la cognición y la cultura. En este sentido, "las personas dan significado a las situaciones en las que participan y a su propia actividad en función de sus características personales, idiosincrásicas, sus ideas, sus conocimientos, su experiencia, sus intereses, etc." (Cubero, \& Luque 1999, p.144). Por lo que, al hablar de desarrollo cultural, es preciso indicar que "las acciones concretas de cada ser humano, enmarcadas en la cultura, tienen lugar en un contexto concreto. Concebido este como un entorno, como lo que rodea, aunque incluyéndolo en su propia definición, como aquello que entrelaza" (Cole, 1999, p. 129).

Al respecto, Rogoff (1993) indica que los elementos del planteamiento sociocultural cambian la propia naturaleza del desarrollo, así como las reorganizaciones que se relacionan con la aparición de nuevas formas de mediación de los procesos psicológicos. Además, se considera que no existe una única clase de desarrollo relevante para la explicación del funcionamiento intelectual humano (Cubero, \& Luque, 1999).

En relación con el papel que tiene la persona que guía la participación, Rogoff (1993) retoma el concepto de Zona de Desarrollo Próximo, establecido por Vygotsky, pero integrándolo en la denominada participación guiada, aspecto de interés para el contexto escolar. 


\section{MATERIAL Y MÉTODO}

El método utilizado en esta investigación se centra en el análisis de contenido, el cual brinda posibilidades de considerar el discurso analizado y cuantificado de los materiales que establecen comunicación humana (Porta, \& Silva, 2003). La metodología permite establecer un trabajo en el cual se reflejan las etapas, en un orden coherente.

\subsection{Procedimiento}

En primer término, se determina la muestra, la cual debe ser adecuada, según los intereses y las particularidades que se requieren para la investigación. Posteriormente, se deben determinar las unidades de análisis y de contexto como sujetos de la observación, para luego establecer la construcción de las categorías (Fernández, 2002).

En este caso, se parte de las políticas públicas establecidas para la incorporación de las TIC en la infancia en el contexto educativo.

\subsection{Población}

En cuanto a la población, esta se refiere a los informes internacionales, regionales y nacionales emitidos por las diversas instancias normativas que registran material pertinente con respecto a la niñez desde preescolar hasta educación primaria para dar respuesta al objetivo planteado.

\subsection{Unidades de análisis}

Las unidades de análisis corresponden a los segmentos expuestos en los mensajes escritos y visuales establecidos en categorías y subcategorías que se derivan de los hallazgos de la investigación y que permiten evidenciar lo expuesto en las políticas públicas sobre las TIC en la población infante.

\subsection{Categorías}

Las categorías de análisis de la investigación son exhaustivas, es decir, abarcan todas las subcategorías posibles que permitan generar un planteamiento fiable de los resultados. Al mismo tiempo, son homogéneas y excluyentes, de manera que las unidades identificadas no responden paralelamente a más de una subcategoría. Acorde con lo anterior, los resultados se exponen en el sistema de categorías.

\section{RESULTADOS}

Los resultados, según los fundamentos referidos a las políticas públicas y elementos que respaldan el uso de las tecnologías en la población de la niñez, están tendientes a guiar el avance de la misma en el proceso educativo mediado por la TIC, como categoría develan una política centrada mayoritariamente en la cobertura donde los esfuerzos apuntan a la colaboración institucional para mejorarla, unida a planes de monitoreo y capacitación que garantice procesos educativos en las escuelas (SITEAL, 2019). Al respecto, es el Estado quien regula esa cobertura, de tal forma que se dictamina dónde enfocar el acceso digital, las regiones de 
un país que tendrán prioridad, el tipo y calidad del recurso tecnológico. La distribución de la infraestructura tecnológica, en ausencia de programas de evaluación y monitoreo, responde a los intereses de los patrocinadores del gobierno. Este acceso así concebido, se plantea como un servicio y no como un derecho lo cual va en detrimento de su democratización. Cabe destacar que sí existe la visión de avanzar a tener conectividad como un derecho que permita la inclusión y educación de calidad para la niñez (Rueda, \& Franco, 2018).

La efectividad de la política en lo que respecta a infraestructura tecnológica se concibe desde su incorporación en los sistemas educativos y el acceso a la tecnología que estos brindan a la niñez. Se enfatiza el acceso, la provisión de equipos y conexiones a internet (Pavez, 2014), ya que los esfuerzos para la creación de infraestructura tecnológica implican el desarrollo de competencias digitales. Estas se impulsan desde el sector educativo el cual se debate internamente entre dar mayor énfasis al acceso o transitar hacia el desarrollo de habilidades digitales, lo cual está supeditado a los gobiernos del momento. (SITEAL, 2019). Un papel fundamental lo desempeña la empresa privada, quien enfatiza sus acciones en la cobertura y, además, realiza colaboraciones o proyectos con instituciones gubernamentales, en los cuales se carece de representación civil e imperan los intereses económicos sobre los objetivos educacionales (Rueda, \& Franco, 2018).

Si bien la política es tendiente a generar cobertura tecnológica, esta no se distribuye equitativamente. La cantidad de recurso tecnológico disponible para cada infante es desigual, y la distribución en las regiones más alejadas o vulnerables aún requiere mayor monitoreo y estructuras evaluadoras para fomentar la inclusión educativa (Cuevas, \& Núñez, 2016). Cabe destacar que al mirar con mayor atención los planes y programas de estudio se visualiza diversas tendencias: el uso de equipo tecnológico y manejo de datos, el enfoque en la utilización de herramientas digitales atomizadas como estrategias didácticas, un énfasis en el aprendizaje, así como esfuerzos para desarrollar la capacidad de resolver problemas (Carneiro et al., 2021).

Las políticas públicas nacionales para el desarrollo de las TIC en la niñez en México y Costa Rica, como categoría, evidencia el énfasis en dotar de bienes y servicios informáticos - acceso a Internet y donación de equipos y dispositivos de cómputo- a la población infantil vulnerable, con la premisa de que se trata de una población que ha sido discriminada por décadas a través de la ejecución de políticas educativas oficiales que centraron su actuación en ciertas partes del sistema educativo, mostrando abandono sobre otras. Como ejemplos, se puede citar la distribución del presupuesto, el cual ha beneficiado a las escuelas urbanas sobre las rurales e indígenas (DOF, 2020).

En consecuencia, el gobierno mexicano se ha propuesto revertir esta tendencia estableciendo la equidad como eje regulador de la acción educativa estableciendo el sistema educativo al servicio de la niñez y adolescencia del país para garantizar aprendizajes significativos con ayuda de las Tecnologías de la Información, Comunicación, Conocimiento y Aprendizaje Digital, garantizando el equipamiento adecuado de los centros educativos para potenciar el máximo logro de los aprendizajes a través del incremento al acceso a Internet en las escuelas.

En el caso de Costa Rica, desde el MEP (2017), se ha implementado como transformación curricular la implementación de una ciudadanía digital en procura de romper la brecha digital, de manera que se orienta a las personas docentes que implementen el uso y aprovechamiento de la tecnología en todos los niveles educativos.; para ello, se trabaja en la expansión solidaria y universal de conectividad.

En relación con las estrategias propuestas en las políticas públicas para la niñez en un contexto tecnológico, en esta categoría, se identifica que buscan apoyar a quienes se encuentran en desventaja. Por ello, establecen algunas estrategias como la denominada Estrategia Nacional de Atención a la Primera Infancia 
y un grupo de programas abocados a redistribuir oportunidades y recurso a través de diferentes tipos de becas en todos los niveles educativos. La finalidad es estipular a la educación como un catalizador para el logro de un desarrollo nacional sostenible.

Por esta razón, se coloca al sistema de educación nacional bajo el dominio del Estado, con la justificación de trasformar estructuralmente dicho sistema, estableciendo como eje central a los educandos por ser los destinatarios finales de la suma de esfuerzos y voluntades. Sin embargo, esta política centralista puede debilitar la toma de decisiones que debería realizar, de manera independiente, cada uno de los 32 estados de México, considerando sus características geográficas, la inversión en el gasto educativo, las particularidades de los educandos y los docentes por niveles educativos, la calidad y cantidad de la infraestructura educativa, los índices educativos, entre muchas más, que diferencian la aplicación de los planteamientos gubernamentales.

Las estrategias enfocadas a la integración de las TIC en la educación de la niñez mexicana aluden al desarrollo de servicios que fortalezcan los aprendizajes regionales y comunitarios, mediante el uso social de las lenguas indígenas y de las tecnologías de la información, comunicación, conocimiento y aprendizaje digital. De igual modo, la procuración de que todas las escuelas de los diferentes tipos, niveles y modalidades cuenten con libros de texto gratuitos, material didáctico y tecnologías de la información, comunicación, conocimiento y aprendizaje digitales diseñados para estudiantes con discapacidad.

Otra de las estrategias desarrolladas para cumplir con este fin es la instrumentación de métodos pedagógicos innovadores, inclusivos y pertinentes, que fortalezcan los procesos de enseñanza y aprendizaje orientados a mejorar la calidad de la educación que reciben las niñez, adolescentes y jóvenes. Por ello, se debe potenciar los métodos de enseñanza del personal docente, mediante el uso pertinente y sostenible de recursos educativos digitales y audiovisuales que fortalezcan los aprendizajes de las niñez y ofrecer talleres creativos e innovadores en áreas relacionadas con las ciencias, la tecnología, las ingenierías, las matemáticas y la robótica; consolidar un ecosistema digital educativo mediante la gestión de una plataforma de contenidos en múltiples formatos - Internet, redes sociales, Red EDUSAT, radio y televisión- y apoyar el acceso y utilización pertinente y sostenible de las TICCAD en los procesos de la vida cotidiana con una perspectiva crítica de los contenidos y materiales disponibles en medios electrónicos, plataformas virtuales y redes sociales (DOF, 2020).

Los procesos educativos potenciadores de la tecnología en la niñez según las políticas públicas, como categoría consideran que la tecnología en la niñez implica una valoración de las habilidades que puedan lograr, tales como respeto, solidaridad, tolerancia hacia los otros, trabajo colaborativo, capacidad reflexiva, creatividad, y la autoexploración en su contacto con las tecnologías de información (Soto, 2017). Una estructura que permite la participación de la niñez con la tecnología son los procesos educativos. Los infantes pueden tener acceso a la tecnología al usar la educación como vehículo para transitar por la carretera digital, aunque el fin primordial es el aprendizaje de un currículo específico. Para ello, las estrategias de acompañamiento a los docentes, monitoreo y evaluación de los aprendizajes se convierten en estructuras institucionales, de tal forma que la escuela se transforma en un ecosistema de comunicación (SITEAL, 2019).

La niñez desarrolla habilidades digitales que les permiten aprender rápidamente el manejo de dispositivos y medios digitales que cambian constantemente su manera de acceso, lograr socializar, ser contactados y escuchados, comunicar e informar sus problemas y hallar soluciones para sí mismos y para otros. Inclusive aprenden nuevas formas de adquirir conocimiento. Establecen relaciones con otros niños en diferentes partes del mundo y realizan intercambios culturales (UNICEF, 2017) por lo que el andamiaje que 
ofrecen los procesos educativos es importante para cada país y la formación de habilidades digitales para sus ciudadanos, principalmente los más pequeños.

La zona de desarrollo próximo, como categoría, juega un papel relevante en los procesos educativos de la niñez, debido a que el apoyo que reciban tanto de las personas adultas, así como de sus pares es fundamental para lograr alcanzar las metas propuestas (Vygotsky, 1978).

En la conceptualización de la ZDP, hay que considerar el binomio que se interrelaciona como lo es el desarrollo de la niñez y su aprendizaje, por lo que se debe tener presente que en la niñez se desarrolla la base para los elementos de salud, de seguridad y de identidad cultural (UNICEF, 2017).

Asimismo, el proceso de andamiaje, en el proceso educativo mediado por las TIC, es imperativo para lograr en la niñez un desarrollo óptimo e integral, puesto que en esta interacción entre un sujeto con experiencia mayor se enfoca en la transformación de la persona, en este caso la niñez (Vygotsky, 1978).

Las prácticas culturales conectadas con los procesos biológicos de la niñez mediante el uso de la tecnología, desde lo propuesto en las políticas públicas, es una categoría que permite visualizar el desarrollo del individuo como un ser biológicamente cultural, por lo que el desarrollo humano se constituye en un proceso cultural. Por consiguiente, aquí el uso de la tecnología es relevante y pertinente, al ingresar al ámbito educativo estableciendo una lógica de reproducción social.

\section{DISCUSIÓN}

Los resultados encontrados generan un punto central para la discusión de las políticas públicas que tienen relación con el uso de las tecnologías en las infancias, centradas en México y Costa Rica, así que en este apartado se resaltan los resultados más destacados apoyando estos con otros autores similares que permitan complementar los obtenidos en esta investigación.

Los gobiernos de los países analizados hacen esfuerzos para que sus políticas respondan a los avances tecnológicos en todos los sectores. Específicamente en la educación, estas repercuten directamente en la cobertura, la adquisición de competencias digitales por parte de docentes y disidentes y la integración tecnológica en las prácticas pedagógicas. En este sentido, se debe trabajar en mayor medida sobre la participación de la niñez al hacer uso de los distintos dispositivos que se tengan en el aula, por lo que es certero considerar los planteamientos de las políticas públicas en las que se indica que es fundamental, enfatizar en prácticas educativas que vinculen las TIC al conocimiento y acciones didácticas y de evaluación (Lion, 2019).

Otro elemento para resaltar es que las políticas públicas abarcan un sentido de políticas de gobierno y no de Estado, que puedan ser sostenidas para que en la práctica se procure un ordenamiento fuerte en cuanto a la consideración del uso correcto de las TIC. Esto es relevante ya que, desde la normativa que se establece en las políticas públicas en favor de la niñez, se abarcan otras áreas como lo es la protección de los derechos, que si bien es cierto se convierte en un elemento relevante, se deja de lado una política robusta en cuanto a que en los centros educativos a los que asisten la niñez esté presente en la mediación de un contexto tecnológico (UNICEF, 2017).

Con respecto al abordaje cultural, Rogoff (2003) indica que es preciso considerar las particularidades de las diferentes comunidades culturales y la relación entre las comunidades individuales y culturales. Por ello, no se debe separar al individuo en un porcentaje de características individuales y culturales ni establecer una dicotomía entre la biología y la cultura, ya que en estas relaciones se desprende un bagaje cultural al considerar las diferentes organizaciones sociales que participan en el desarrollo social y aquí está presente el proceso educativo iniciando desde la niñez, en un contexto tecnológico. 
Es relevante destacar el binomio ineludible de educación y tecnología que implica necesariamente la participación guiada de la educación infantil. Por ello, es necesario desarrollar estrategias para que los cuidadores de la niñez y docentes puedan mediar positivamente entre el mundo digital y la niñez. En este caso, la capacitación específica en la infancia también permite el cuidado entre pares lo que resulta en formas más efectivas de protección (UNICEF, 2017).

\section{CONCLUSIONES}

Las principales conclusiones del estudio se centran en dar un enfoque de lo que el análisis de contenido ha permitido, al visualizar la realidad de las políticas públicas en función del uso de las tecnologías en la población estudiantil de las infancias, en Costa Rica y México Es así como se evidencia que los gobiernos han trabajado en la implementación del uso de las tecnologías desde los diversos niveles, incluyendo las infancias que asisten a los centros educativos de preescolar y educación primaria. Sin embargo, la cobertura es el fin primordial, dejando de lado procesos de seguimiento y control.

Asimismo, las políticas públicas desde su fundamentación deben potenciar el uso de las tecnologías, como generadoras del desarrollo de las habilidades en las prácticas de aula. Además, es de suma relevancia que en los procesos educativos de la niñez se considere la ZDP, para que se pueda lograr una formación con el apoyo de las personas docentes y de sus pares que están con mayor desarrollo en la tecnología.

El planteamiento de las políticas públicas debe ser llevado a la práctica en las aulas, y que la niñez tenga una mayor participación, de manera que se trascienda a la enseñanza tradicional. Por tanto, se debe valorar que se trabaje en procura de considerar a toda la población, y desligar que las TIC están enfatizadas a la niñez vulnerable.

En función de hacer evidente las políticas públicas en el ámbito educativo de la niñez, es preciso generar actividades en las cuales la infancia pueda ser participe en la construcción de aprendizajes.

En relación con la promulgación de políticas públicas es preciso que se considere las realidades de los espacios, por lo que se debe promover escenarios educativos y que las TIC sean un pilar fundamental para que se continúe en el avance tecnológico que se visualiza cada día en la sociedad.

En síntesis, es necesario que las políticas públicas deben dejar atrás ese sentir populista, para que la integración de las TIC permita un avance en los procesos de educación de la niñez, y que esta población desarrolle las habilidades según el nivel de educación en el cual se encuentre. Si bien es cierto, hay evidencias de elementos de políticas públicas hace falta una conexión con la TIC y con la niñez, ya que no están del todo articulados en Costa Rica y México, por lo que falta por hacer en ambos países.

\section{REFERENCIAS}

Adrea, A. (2020). La educación infantil en la era de las TIC en Nigeria: un fenómeno sociocultural tripartito. AYER JOURNAL, 7(3), 82-95. https://doi.org/10.1445/ayerjournal.v27i3.117

Briceño. l., Flórez, R., \& Gómez, D. (2019). Usos de las TIC en preescolar: hacia la integración curricular. Revista Panorama, 13 (24), 21-32. http://dx.doi.org/10.15765/pnrm.v13i24.1203
Bruner, J. (1997). La educación, puerta de la cultura. Editorial Visor.

Cabero, J. (2007). Las necesidades de las TIC en el ámbito educativo: oportunidades, riesgos y necesidades. Revista Tecnologías y Comunicación Educativas, 21(45), 4-19.

Calderón, M., Padilla, M., \& Fornaguera, J. (2013). Introducción de las tecnologías en el aula de dos preescolares públicos costa- 
rricenses: Estrategias de autogestión, alcances y limitaciones. Revista Electrónica Actualidades Investigativas en Educación. 13(2), $1-23$

Cole, M. (1999). Psicología Cultural. Una disciplina del pasado y del futuro. Ediciones Morata.

Coll, C. (2009). Aprender y enseñar con las TIC: expectativas, realidad y potencialidades. En R. Caneiro, J. Toscano, y T. Díaz Coord. (Eds.), Los desafíos de las TIC para el cambio educativo. Colección Metas Educativas (p. 113-126). OEI/ Fundación Santillana.

Cubero, R., \& Luque, A. (2001). Desarrollo, educación y educación escolar: la teoría sociocultural del desarrollo y del aprendizaje. En J. Palacios, A. Marchesi y C. Coll (Comps.). Vol. I: Psicología Evolutiva (2. ${ }^{a}$ Edición) Desarrollo psicológico y educación (pp. 137-156). Alianza Editorial.

Cuevas, F., \& Núñez, N. (2016). Tecnologías digitales y Educación. Prosic Universidad de Costa Rica, 9, 373-402

Diario Oficial de la Federación (DOF) (2020). Programa Sectorial de Educación 2020-2024. Gobierno de México. https:// www.dof.gob. $\mathrm{mx} /$ nota detalle.php? codigo $=5596202 \& \mathrm{fec}$ $\underline{\mathrm{ha}=06 / 07 / 2020}$

Diaz, T, Carneiro, R., \& Toscano, J. (2018). Los desafíos de las Tic para el cambio educativo. AECID

Duek, C., \& Benítez, S. (2018). Infancias y tecnologías en Argentina: interacciones y vínculos intergeneracionales. Revista Nómadas, (49), 121-135. https://doi.org/10.30578/nómadas. n49a7

El diálogo (2019). México. Informe del progreso de política de primera infancia. https://www.thedialogue.org/analysis/mexico-informe-de-progreso-de-politicas-de-primera-infancia/

Espinoza, L., \& Rodríguez, R. (2017). El uso de tecnologías como factor del desarrollo socioafectivo en niños y jóvenes estudiantes en el noroeste de México. RICSH Revista Iberoamericana de las Ciencias Sociales y Humanística, 6(11), 151-170. https://doi.org/10.23913/ricsh.v6i11.113

Fernández, F. (2002). El análisis de contenido como ayuda metodológica para la investigación. Revista Ciencias Sociales, (96), 35-53.

Fondo de las Naciones Unidas para la Infancia. (UNICEF). (2017). Estado mundial de la infancia. Niños en un mundo digital. https://www.unicef.org/media/48611/file
Fondo de las Naciones Unidas para la Infancia. (UNICEF). (2017). Política para la primera infancia 2015-2020. Consejo de la Niñez y la Adolescencia. http://www.ddc.mep.go.cr/sites/all/files/ddc mep go cr/archivos/politica para la primera infancia 2015-2020.pdf

Fondo de las Naciones Unidas para la Infancia. (UNICEF) (2014). Los derechos de la infancia en la era digital. Chile. www.unicef. org/lac/library 6188.htm

Instituto Nacional para la Evaluación de la Educación. (2018.) La política educativa de México desde una perspectiva regional. https://www.buenosaires.iiep.unesco.org/sites/default/files/archivos/LaPoliticaEducativaRegional.pdf

Lion, C. (2019). Los desafíos y oportunidades de incluir tecnologías en las prácticas educativas. Análisis de casos inspiradores. Análisis comparativos de políticas de educación y tic. IIPEUNESCO. https://www.buenosaires.iiep.unesco.org/sites/ default/files/actividades/2019-09/An\%C3\%A1lisis\%20comparativos\%20-\%20Pol\%C3\%ADticas\%20TIC\%20-\%20Carina\%20Lion 0.pdf

Ministerio de Educación Pública (MEP) (2017). La política educativa. Consejo Superior de Educación. 1-28 https://www.mep. go.cr/sites/default/files/page/adjuntos/politicaeducativa.pdf

Pavez, M. (2014). Los derechos de la infancia en la era de internet. América Latina y la Nuevas Tecnologías. UNICEF

Porta, L., \& Silva, M. (2003). La investigación cualitativa: El Análisis de Contenido en la investigación educativa. Anuario Digital de Investigación Educativa, 14, 1-18.

Rodríguez, A., Hinojo, F., \& Ágreda, M. (2019). Diseño e implementación de una experiencia para trabajar la interculturalidad en Educación Infantil a través de realidad aumentada y códigos QR. Educar, 55(1), 59-77.https://www.raco.cat/index. php/Educar/article/view/348881

Rogoff, B. (1993). Aprendices del pensamiento: el desarrollo cognitivo en el contexto social. La participación guiada y la transferencia de la responsabilidad en el control de las actividades. Editorial Paidós.

Rogoff, B. (2003). The cultural nature of human development. Oxford University Press.

Rueda, R., \& Franco, M. (2018). Políticas educativas de tic en Colombia: entre la inclusión digital y formas de Resistencia 
trans- formación social. Revista Pedagogía y Saberes, (48), 9-25. https://doi.org/10.17227/pys.num48-7370

Ruiz, M., \& Hernández, V. (2018). La incorporación y uso de las TIC en la Educación Infantil. Un estudio sobre la infraestructura, la metodología didáctica y la formación del profesorado en Andalucía. Píxel-Bit. Revista de Medios y Educación (52), 8196.https://doi.org/10.12795/pixelbit.2018.i52.06

Siraj, J., \& Romero, R. (2017). De la aplicación a la participación activa de las TIC en Educación Infantil. Píxel-Bit. Revista de Medios y Educación, (51), 65-81. http://dx.doi.org/10.12795/ pixelbit.2017.i51.11

Sistema de Información de Tendencias Educativas en América Latina (SITEAL) (2019). Educación y TIC. UNESCO.https://siteal. iiep.unesco.org/sites/default/files/sit informe pdfs/siteal educacion y tic 20190607.pdf

Smith, M. (1991). Formación de la personalidad desde una perspectiva cultural y dinámica de la personalidad en situaciones de cambio. Editorial Universidad de Costa Rica.

Soto, J. (2007). Políticas educativas y nuevos contextos de intervención en relación con las Tic. Panorama actual en el ámbito europeo y español. Revista de Investigación en Educación, 4(1), 4-21.

Vygotsky, L. (1978). El desarrollo de los procesos psicológicos superiores. Editorial Crítica. 